\title{
Vision-guided Music Source Separation via a Fine-grained Cycle-Separation Network (Supplementary Materials)
}

Anonymous Author(s)

Submission Id: 2072

\section{ACM Reference Format:}

Anonymous Author(s). 2021. Vision-guided Music Source Separation via a Fine-grained Cycle-Separation Network (Supplementary Materials). In ACMMM '21: ACM Multimedia, October, 2021, Chengdu, China. ACM, New York, NY, USA, 5 pages. https://doi.org/10.1145/1122445.1122456

\section{A RESULTS OF MULTI-SOURCE SEPARATION (MIXTURE OF SOLOS AND DUETS)}

Table 1 shows source separation results of multi-source separation in the MUSIC dataset. In the experiment, we train our FCSN model using mixtures of solos and duets, multi-source videos. Here, solos refer to videos containing single musical instruments, and duets are videos containing two musical instruments. We compare our results with approaches of NMF [4], SOP [6] and Co-Separation [1] As shown in Table 1, the proposed FCSN approach achieves better separation performance.

\begin{tabular}{lcc}
\hline \multirow{2}{*}{ Method } & \multicolumn{2}{c}{ multi-source } \\
\cline { 2 - 3 } & SDR & SIR \\
\hline NMF [4] 2007 & 0.92 & 5.68 \\
SOP [6] 2018 & 6.05 & 9.81 \\
Co-Separation [1] 2019 & 7.64 & 13.8 \\
\hline Ours & $\mathbf{8 . 5 5}$ & $\mathbf{1 4 . 1 3}$ \\
\hline
\end{tabular}

Table 1: Results of multi-source separation in the MUSIC. In the experiment, videos containing solos and duets musical instruments are used for model training. Obviously, the proposed FCSN approach outperforms existing baselines.

\section{B RESULTS OF HAND DETECTION AND OBJECT SCREENING}

The object detector is trained on about 30k images of 15 object categories from Open Image dataset [2] $]^{1}$. The 15 categories include

\footnotetext{
${ }^{1}$ https://github.com/jwyang/faster-renn.pytorch

Permission to make digital or hard copies of all or part of this work for personal or classroom use is granted without fee provided that copies are not made or distributed for profit or commercial advantage and that copies bear this notice and the full citation on the first page. Copyrights for components of this work owned by others than ACM must be honored. Abstracting with credit is permitted. To copy otherwise, or republish, to post on servers or to redistribute to lists, requires prior specific permission and/or a fee. Request permissions from permissions@acm.org.

ACMMM '21, October, 2021, Chengdu, China

(C) 2021 Association for Computing Machinery.

ACM ISBN 978-1-4503-XXXX-X/21/07 . .\$15.00

https://doi.org/10.1145/1122445.1122456
}

Banjo, Cello, Drum, Guitar, Harp, Harmonica, Oboe, Piano, Saxophone, Trombone, Trumpet, Violin, Flute, Accordion, and Horn. The hand detector [5] is pre-trained in Oxford Hand dataset [3], which is a lightweight model. It can quickly detect hands and their orientations in images.

With detected hand and musical instrument candidates, we further design a screening method to correctly determine critical musical instruments. In each video, the object detector detects instruments in several frames and selects candidates with the highest confidence. The hand detection also follows the same rule, selecting hands with the highest confidence. With detected hands and their orientations, we perform object screening on detected object templates to build correct correspondences between objects and sound sources. The overall screening operation is shown in Fig. 1. The screening operation is performed in two steps. Firstly, we select reliable objects from all detected templates by setting a threshold of 0.9 on the prediction probabilities. Secondly, we calculate the distance $d_{i j}$ from the hand $h_{j}$ to the object $O_{i}$, and calculate the angle $\alpha_{i j}$ between the orientation of hand $h_{j}$ and the distance $d_{i j}$. Since the distance is more important in the determination of object selection, we sort distances and select objects which are nearest to detected hands. Following that, we further check whether the angle $\alpha_{i j}$ is reasonable. The angle $\alpha_{i j}$ should be within a constant range, an angle threshold of $120^{\circ}$ is set in our experiments. The screening operation helps to finally select reliable object candidates to assist the music source separation.

We illustrate several results of screening objects in Fig. 2. It can be seen that hand detection helps to distinguish real vocal instruments and background instruments. Therefore, the operation of hand detection based screening operation contributes to correct vision representation in our proposed approach.

\section{VISUALIZATION OF SEPARATION RESULTS}

Fig. 3 and Fig. 5 visualize separation results of 2-mix, 3-mix and real mixture experiments in the MUSIC dataset. The visualization includes detected object regions, mixed audio spectrograms, and separated sound spectrograms. We compare the separation results of our FCSN approach with the Co-separation approach [1] in the figure. We use yellow circles and blue rectangles to mark local regions where we compare our FCSN approach with the Co-separation approach [1]. It can be observed that our results are more similar to the ground truth. The visualization demonstrates that our FCSN approach achieves better separation results. 

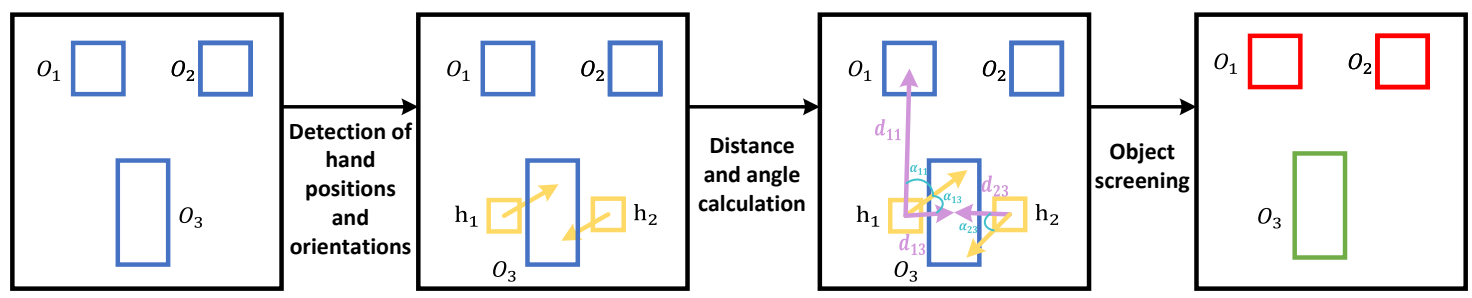

Figure 1: The detailed object screening operation with the assistance of hand positions and orientations. Here, $O_{i}$ is the $i t h$ detected object, marked by blue boxes. And $h_{j}$ refers to the $j t h$ detected hand, where we use a yellow box and a yellow arrow to represent the position and orientations of hands. We use $d_{i j}$ to describe the connection from the hand $h_{j}$ to the object $O_{i}$, and use $\alpha_{i j}$ to represent an angle between the orientation of hand $h_{j}$ and $d_{i j}$. Through the object screening, selected objects are marked with green boxes, and objects with red boxes are abandoned.

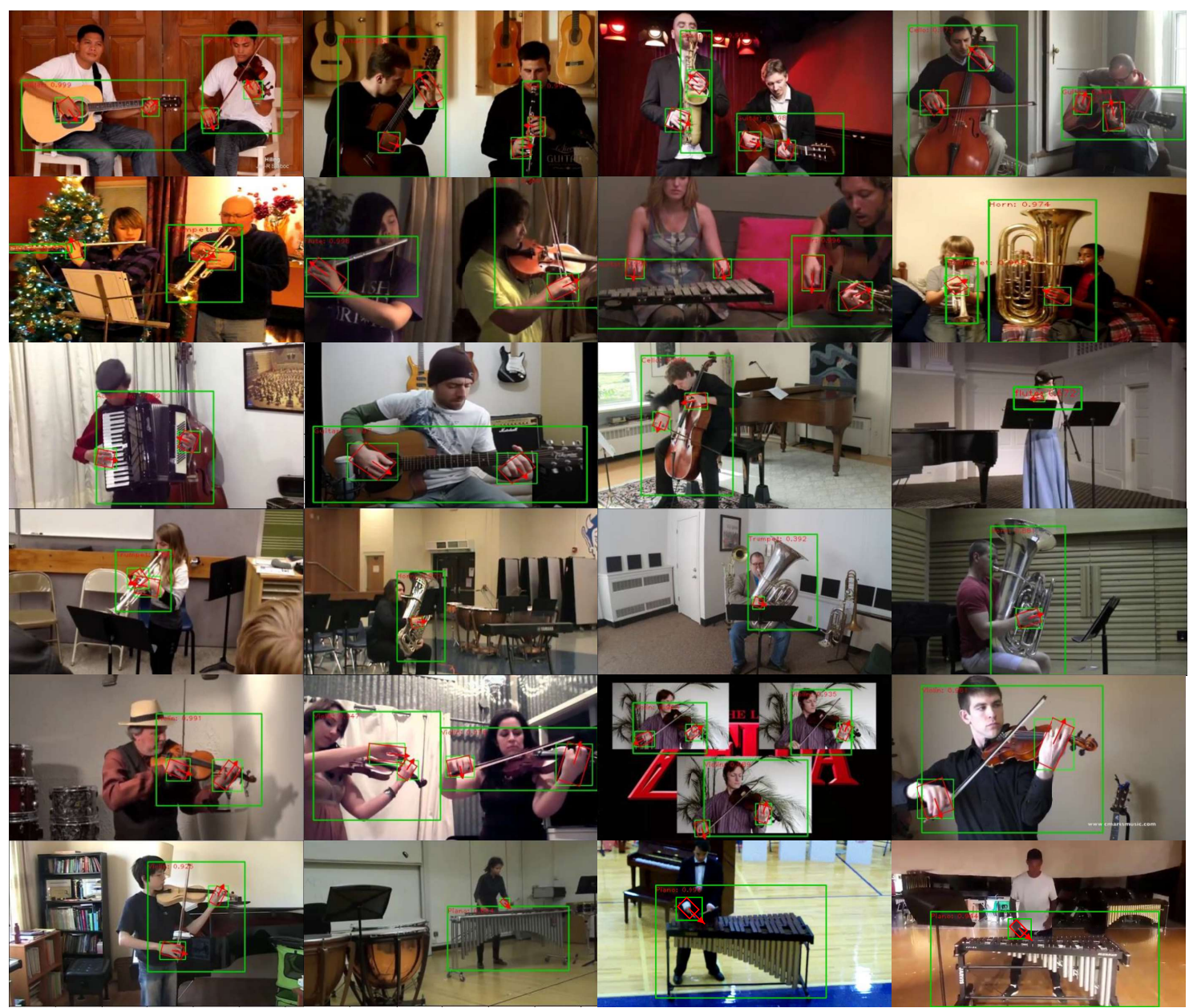

Figure 2: Results of hand detection and object screening. The green boxes localize detected instruments and hands. The red boxes and the red arrows represent estimated hand orientations.

\section{ITERATION TIMES FOR CYCLE SEPARATION}

We perform 2-mix experiments to search for optimal iteration times in three datasets, the MUSIC, the AudioSet, and the VGGSound.
We record the separation results of each iteration and draw these results on Figure 4. As shown, with the increase of iteration times, the $\mathbb{E}\left[S_{l e f t}\right]$ gradually decreases and reaches the lowest point at the 


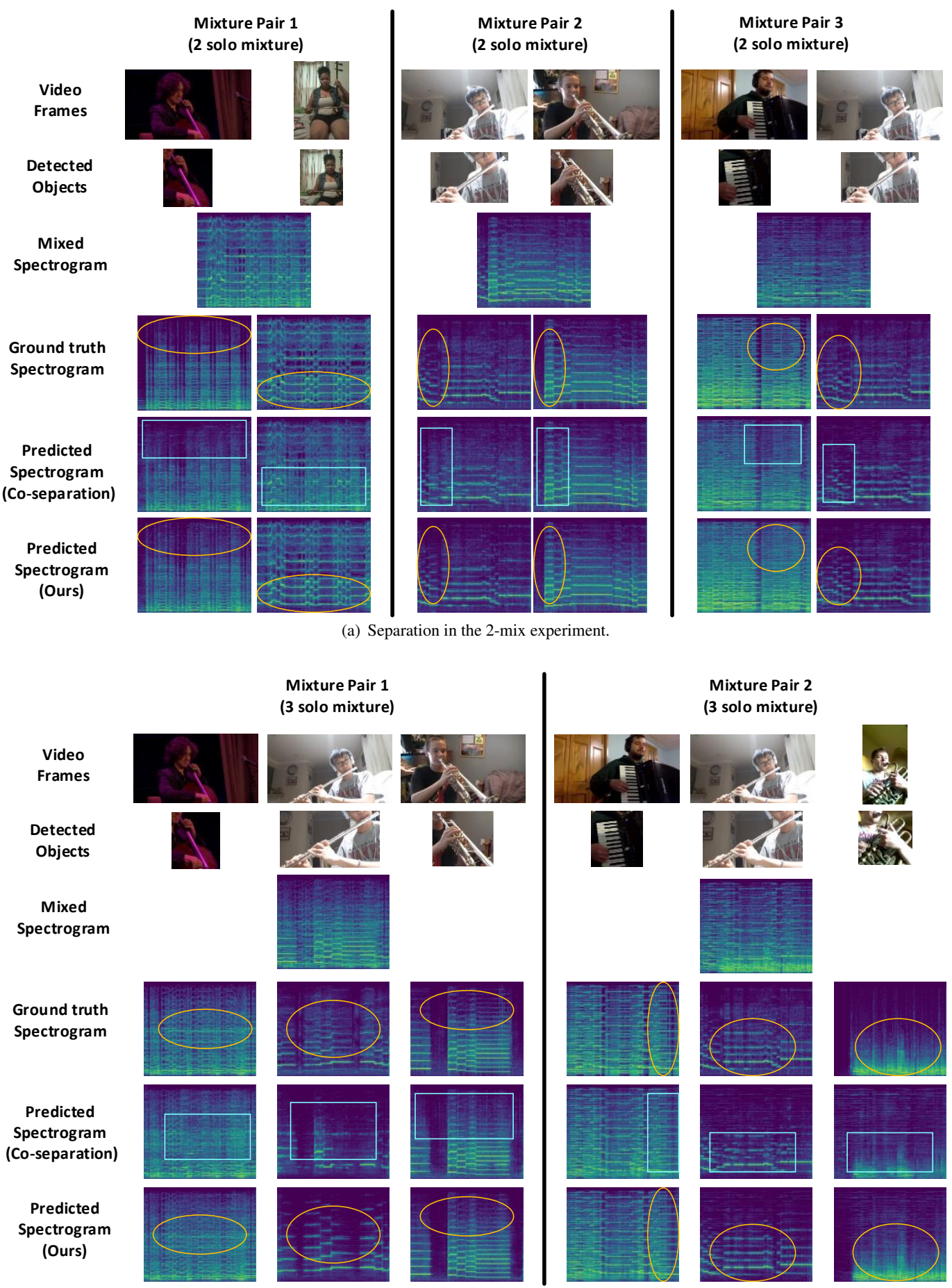

(b) Separation in the 3-mix experiment

Figure 3: Visualization on separation results of 2-mix and 3-mix experiments in the MUSIC dataset. We compare the results of our FCSN approach with the Co-separation approach [1]. Fig. 3(a) shows results of 2-mix experiment, and Fig. 3(b) illustrates results of 3-mix experiment. The orange circles indicate that our results are more similar to the ground truth spectrogram, and the blue rectangles illustrate that results of the Co-separation approach are different from the ground truth. It demonstrates the effectiveness of our FCSN approach. 


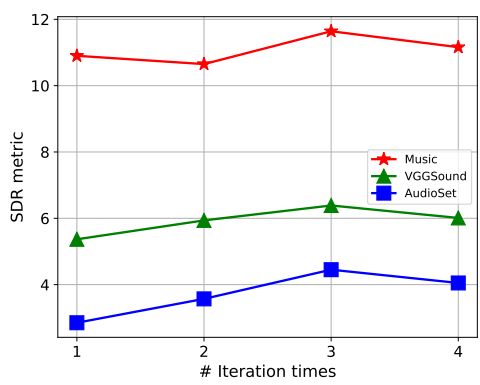

(a) SDR

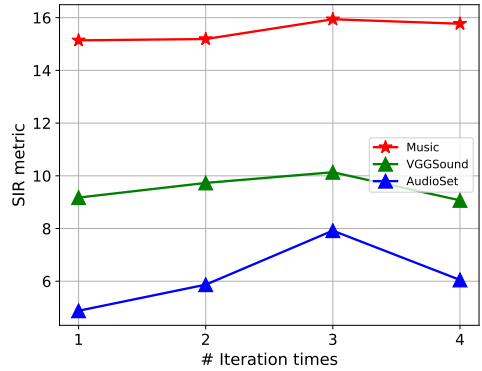

(b) SIR

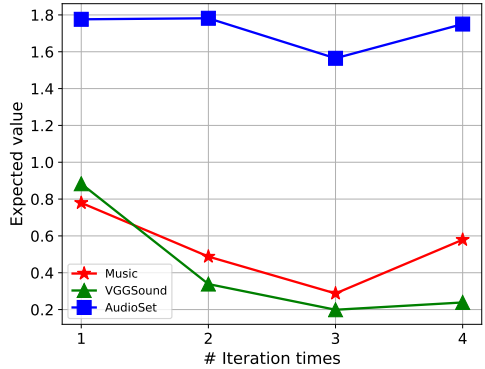

(c) Expected value of the residual spectrogram

Figure 4: Evaluation results of the cycle separation of our FCSN model with different iteration times in three datasets, MUSIC, VGGsound, and AudioSet. We record the SDR, SIR accuracies, and expected values of the residual spectrogram, $\mathbb{E}\left[S_{l e f t}\right]$, for each iteration. The red, green, and blue curves respectively stand for Music, VGGsound, and AudioSet. Results demonstrate that our FCSN achieves best separation results after 3 iteration times in three datasets.

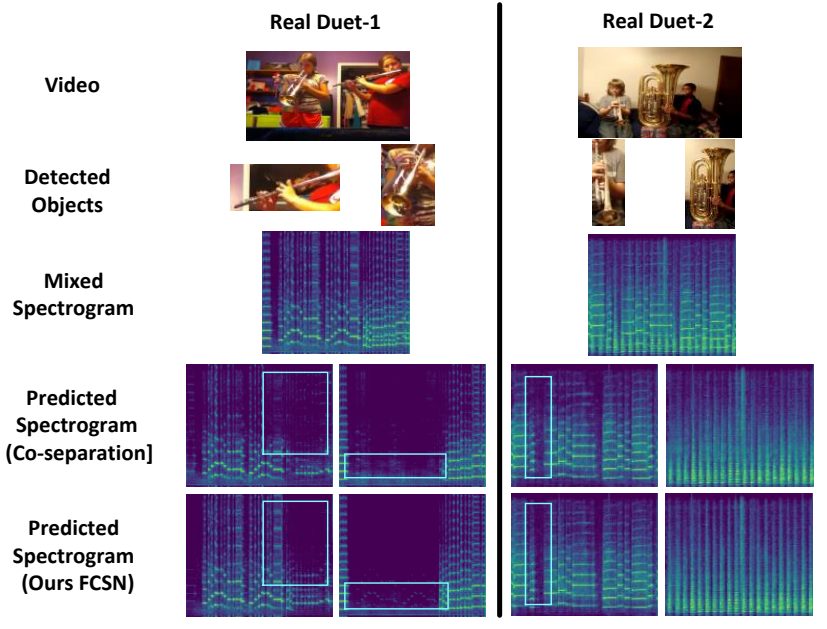

Figure 5: Visualization of separation results in real mix videos containing duets. Separation spectrograms obtained using the "Co-separation" approach [1] and our FCSN model are compared. We use the cyan rectangles to figure out region differences. It can be observed that our FCSN model can efficiently separate music sources of different instruments, obtaining reasonable separated spectrograms.

3rd iteration. Correspondingly, the separation accuracies (SDR/SIR) achieve the highest value at the 3rd iteration in three datasets.

\section{E CONTRIBUTION OF CYCLE SEPARATION.}

One major contribution of our FCSN approach is the cycle separation. To demonstrate its effectiveness, we compare the separation results obtained in the preliminary separation step with the results obtained after cycle separation operation by the proposed FCSN approach. The 2-mix experiment is performed in the MUSIC dataset. The visualization of separation results is shown in Figure 6. Compared with the ground truth, it can be seen that the preliminary separation

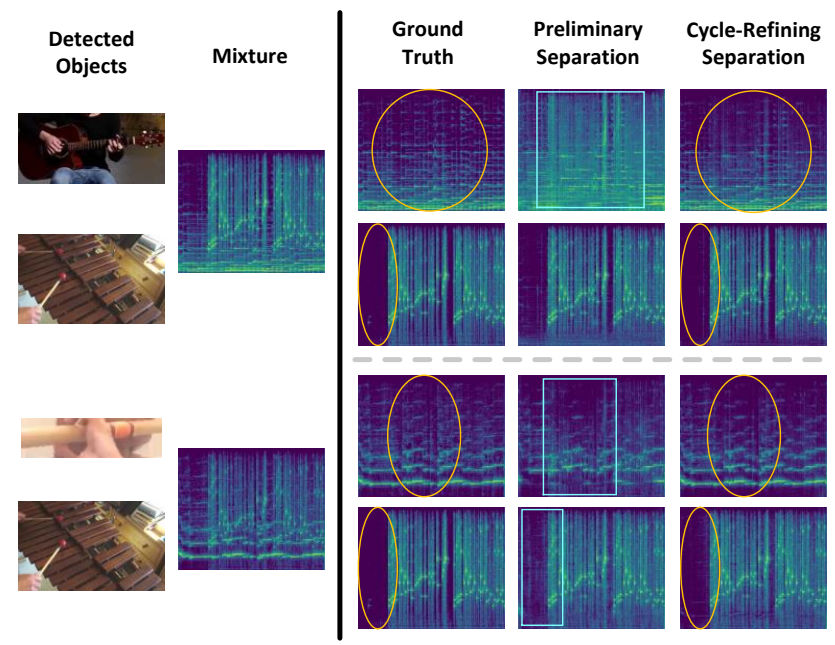

Figure 6: Visualization of separation results of two steps, $i . e$. the preliminary separation and the cycle separation (FCSN). Here, the optimal iteration number is $\mathbf{3}$ in the cycle separation. Obviously, after the refining operation of cycle separation, the separation quality is significantly improved. We use yellow circles to mark the regions where spectrograms are correctly separated by the cycle separation, and use cyan rectangles to figure out incorrect separation results in preliminary separation.

results contain many error parts, while the refined separation results are closer to the ground truth. It certifies that the cycle separation operation significantly improves the performance. Therefore, the cycle separation is effective for music source separation.

\section{REFERENCES}

[1] Ruohan Gao and Kristen Grauman. 2019. Co-separating sounds of visual objects. In $I C C V$.

[2] Alina Kuznetsova, Hassan Rom, Neil Alldrin, Jasper R. R. Uijlings, Ivan Krasin, Jordi Pont-Tuset, Shahab Kamali, Stefan Popov, Matteo Malloci, Alexander Kolesnikov, Tom Duerig, and Vittorio Ferrari. 2020. The Open Images Dataset V4: 
Unified image classification, object detection, and visual relationship detection at scale. IJCV 28, 7 (2020), 1956-1981.

[3] Arpit Mittal, Andrew Zisserman, and Philip H. S. Torr. 2011. Hand detection using multiple proposals. In $B M V C$.

[4] T. Virtanen. 2007. Monaural sound source separation by nonnegative matrix factorization with temporal continuity and sparseness criteria. IEEE Transactions on Audio, Speech, and Language Processing 15, 3 (2007), 1066-1074.

[5] Li Yang, Zhi Qi, Zeheng Liu, Shanshan Zhou, Yang Zhang, Hao Liu, Jianhui $\mathrm{Wu}$, and Longxing Shi. 2018. A light CNN based method for hand detection and orientation estimation. In $I C P R$

[6] Hang Zhao, Chuang Gan, Andrew Rouditchenko, Carl Vondrick, Josh H. McDermott, and Antonio Torralba. 2018. The sound of pixels. In ECCV. 